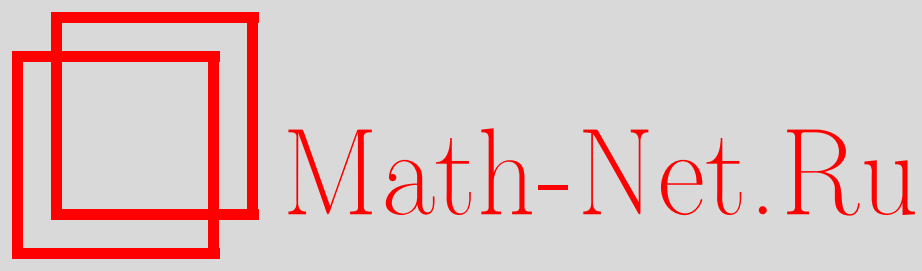

С. Д. Лошкарёв, Вычисление количества решений одного разностного уравнения, Дискрет. матем., 2014, том 26, выпуск 2, 42-57

DOI: https://doi.org/10.4213/dm1279

Использование Общероссийского математического портала Math-Net.Ru подразумевает, что вы прочитали и согласны с пользовательским соглашением http://www.mathnet.ru/rus/agreement

Параметры загрузки:

IP : 52.205 .19 .152

26 апреля 2023 г., 12:38:41

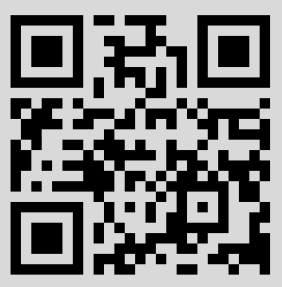




\title{
Вычисление количества решений одного разностного уравнения
}

\author{
() 2014 г. С. Д. Лошкарёв*
}

В алгоритмах хэш-функций семейства $M D x$ используются циклические сдвиги, примитивные булевы функции и прибавления констант. До настоящего момента опубликовано крайне мало работ, пытающихся объяснить, как выбор констант, сдвигов и булевых функций влияет на криптографические свойства алгоритмов.

Г. А. Карпунин и Т. Х. Нгуен предложили модель, в которой устойчивость к дифференциальному криптоанализу можно оценить количественно посредством вычисления количества решений уравнения специального вида.

В настоящей работе в рамках этой модели выведено уравнение для хэш-функции MD5. Трудоемкость анализа одной булевой функции и одного значения циклического сдвига при полном переборе составляет $2^{128}$ операций вычисления шага хэш-функции. В настоящей работе предложены формулы, позволяющие сократить трудоемкость анализа до $2^{44}$ арифметических операций.

Ключевые слова: криптоанализ, криптография, хэш-функция, MD5, дифференциальный криптоанализ, дифференциальная характеристика.

\section{1. Введение}

Одним из наиболее распространенных методов криптоанализа хэш-функций является дифференциальный криптоанализ, изначально предложенный для атак на блоковые шифры Э. Бихамом и Э. Шамиром в 1990 в работе [1]. С использованием метода дифференциального криптоанализа были построены коллизии для хэш-функций MD4, MD5, SHA-1 и других.

Определение 1. Дифференциалъная характеристика - это набор разностей, содержащий разность между исходными сообщениями, разность между значениями хэш-функции для этих сообщений и, возможно, разности между некоторыми промежуточными значениями, получаемыми в процессе вычисления хэш-функции.

При проведении дифференциального криптоанализа осуществляется поиск дифференциальных характеристик, с высокой вероятностью появления. В настоящий момент не существует универсального эффективного метода поиска дифференциальных характеристик с большими вероятностями, равно как не существует универсального эффективного метода вычисления вероятности появления выбранной дифференциальной характеристики.

*Место работы: МГУ им. М.В. Ломоносова, e-mail: loshkaryov@gmail.com 
В работе [2] предложен подход к дифференциальному криптоанализу как к угадыванию решения разностного уравнения, построенного по уравнению, описывающему алгоритм вычисления хэш-функции, с учетом выбранной дифференциальной характеристики. В этой же работе получены формулы, позволяющие вычислять вероятность угадывания решения уравнения для хэш-функции $M D 4$.

Разностное уравнение для MD5 отличается от уравнения, полученного в работе [2], наличием циклических сдвигов. Данное изменение делает вычисление вероятности угадывания решения уравнения существенно более сложным.

Методом полного перебора данную задачу невозможно решить на текущем этапе развития вычислительной техники, так как сложность реализации составит $2^{128}$ операций вычислений шага хэш-функции.

В данной работе представлен метод, позволяющий вычислить данную вероятность со сложностью не более $2^{44}$ арифметических операций.

\section{2. Разностное уравнение для хэш-функции MD5}

Хэш-функция MD5 описана в работе Р. Ривеста [3]. Вычисление функции сжатия хэш-функции может быть описано следующей системой уравнений:

$$
\left\{\begin{array}{l}
Q_{-3}=A ; \\
Q_{0}=B ; \\
Q_{-1}=C ; \\
Q_{-2}=D ; \\
\left(w_{1}, \ldots, w_{64}\right)=\text { MessageExpansion }\left(M_{1}, \ldots, M_{16}\right) ; \\
Q_{j}=\left(\left(f_{j}\left(Q_{j-1}, Q_{j-2}, Q_{j-3}\right) \boxplus Q_{j-4} \boxplus w_{j} \boxplus T_{j}\right)<<<s_{j}\right) \boxplus Q_{j-1} ; \\
\widetilde{A}=Q_{-3} \boxplus Q_{61} ; \\
\widetilde{B}=Q_{0} \boxplus Q_{64} ; \\
\widetilde{C}=Q_{-1} \boxplus Q_{63} ; \\
\widetilde{D}=Q_{-2} \boxplus Q_{62} ;
\end{array}\right.
$$

где $A, B, C, D, M_{i}, w_{i} \in\{0,1\}^{32}, H=A\|B\| C \| D$, MessageExpansion - операция расширения сообщения, $f_{j}:\{0,1\}^{3} \rightarrow\{0,1\}$ - булева функция, применяемая побитово, $A \boxplus B$, где $|A|=T,|B|=R, T \geqslant R$ (через $|A|$ обозначена длина вектора $A$ ) - здесь и далее сложение чисел по модулю $2^{T}$. Значение $\tilde{H}=\tilde{A}\|\tilde{B}\| \tilde{C} \| \tilde{D}$ является результатом вычисления функции сжатия $M D 5_{c}(H, M)$. Также будем пользоваться обозначением $A \boxminus B$, где $|A|=T,|B|=R, T \geqslant R$ - вычитание чисел по модулю $2^{T}$.

Применим общий подход работы [2] к хэш-функции MD5. Bсе обозначения в данном разделе взяты из работы [2].

Выделим из системы (1) уравнение, осуществляющее основное преобразование:

$$
Q_{j}=\left(\left(f_{j}\left(Q_{j-1}, Q_{j-2}, Q_{j-3}\right) \boxplus Q_{j-4} \boxplus w_{j} \boxplus T_{j}\right)<<<s_{j}\right) \boxplus Q_{j-1} ;
$$

Добавим разностное уравнение:

$$
\left\{\begin{aligned}
Q_{j}=\left(\left(f_{j}\left(Q_{j-1}, Q_{j-2}, Q_{j-3}\right) \boxplus Q_{j-4} \boxplus w_{j} \boxplus T_{j}\right)<<<s_{j}\right) \boxplus Q_{j-1} ; \\
Q_{j} \boxplus \Delta Q_{j}=\left(\left(f_{j}\left(Q_{j-1} \boxplus \Delta Q_{j-1}, Q_{j-2} \boxplus \Delta Q_{j-2}, Q_{j-3} \boxplus \Delta Q_{j-3}\right) \boxplus\right.\right. \\
\left.\left.\boxplus Q_{j-4} \boxplus \Delta Q_{j-4} \boxplus w_{j} \boxplus \Delta w_{j} \boxplus T_{j}\right)<<<s_{j}\right) \boxplus Q_{j-1} \boxplus \Delta Q_{j-1}
\end{aligned}\right.
$$


Теперь вычтем из второго уравнения первое:

$$
\left\{\begin{array}{c}
Q_{j}=\left(\left(f_{j}\left(Q_{j-1}, Q_{j-2}, Q_{j-3}\right) \boxplus Q_{j-4} \boxplus w_{j} \boxplus T_{j}\right)<<<s_{j}\right) \boxplus Q_{j-1} ; \\
\Delta Q_{j}=\left(\left(\left(f _ { j } \left(Q_{j-1} \boxplus \Delta Q_{j-1}, Q_{j-2} \boxplus \Delta Q_{j-2}, Q_{j-3}\right.\right.\right.\right. \\
\left.\left.\left.\boxplus \Delta Q_{j-3}\right) \boxplus Q_{j-4} \boxplus \Delta Q_{j-4} \boxplus w_{j} \boxplus \Delta w_{j} \boxplus T_{j}\right)<<<s_{j}\right) \boxminus \\
\left.\boxminus\left(\left(f_{j}\left(Q_{j-1}, Q_{j-2}, Q_{j-3}\right) \boxplus Q_{j-4} \boxplus w_{j} \boxplus T_{j}\right)<<<s_{j}\right) \boxplus \Delta Q_{j-1}\right) .
\end{array}\right.
$$

Введем обозначения $X_{4}=Q_{j-4} \boxplus w_{j} \boxplus T_{j}, A_{4}=\Delta Q_{j-4} \boxplus \Delta w_{j}, s=s_{j}, A_{0}=$ $\left(\Delta Q_{j} \boxminus \Delta Q_{j-1}\right)>>>s, f_{j}=f, X_{1}=Q_{j-1}, X_{2}=Q_{j-2}, X_{3}=Q_{j-3}, A_{1}=\Delta Q_{j-1}$, $A_{2}=\Delta Q_{j-2}, A_{3}=\Delta Q_{j-3}$. Тогда второе уравнение в последней системе примет вид:

$$
\begin{aligned}
\left(f\left(X_{1} \boxplus A_{1}, X_{2} \boxplus A_{2}, X_{3} \boxplus A_{3}\right) \boxplus X_{4} \boxplus A_{4}\right)<<<s= \\
=A_{0}<<<s \boxplus\left(f\left(X_{1}, X_{2}, X_{3}\right) \boxplus X_{4}\right)<<<s .
\end{aligned}
$$

В этом уравнении $X_{i}$ - неизвестные, $A_{i}$ - параметры, $s-$ фиксированная величина.

Следует отметить, что при исследовании хэш-функции $M D 4$ в работе [2] было получено уравнение

$$
f\left(X_{1} \boxplus A_{1}, X_{2} \boxplus A_{2}, X_{3} \boxplus A_{3}\right)=A_{0} \boxplus f\left(X_{1}, X_{2}, X_{3}\right)
$$

Использование циклического сдвига в хэш-функции MD5 не позволяет использовать для данной хэш-функции методы анализа, предложенные в [2] для хэш-функции $M D 4$.

В [2] показано, что если рассмотреть две разные булевы функции $f$ и $g$ и соответствующие им системы вида (1), то соотношение между вероятностями угадывания решения систем будет тем же, что и соотношение между вероятностями угадывания решения уравнения (2).

Итак, требуется вычислить вероятность угадывания решения уравнения (2), выбирая значения переменных $X_{i}$ случайно и равновероятно.

В случае хэш-функции MD5 циклический сдвиг $s$ может принимать любое значение из множества $\{4,5,6,7,9,10,11,12,14,15,17,20,21,22,23\}$.

\section{3. Понятие переноса и связанные с ним факты}

Следуя работе [2], введем некоторые обозначения. Пусть $X=X_{n-1}\|\ldots\| X_{0}$ $\left(X_{i} \in\{0,1\}, i=\overline{0, n-1}\right)$ - двоичный вектор некоторой длины $n$. Нумерация битов осуществляется справа налево. Тогда через $X^{\left[i_{1}, \ldots, i_{k}\right]}, k \leqslant n$, обозначим вектор, равный конкатенации битов вектора $X$ с соответствующими номерами, то есть $X_{i_{k}}\|\ldots\| X_{i_{1}}$. Через $X^{[<T]}, T \leqslant n$ будем обозначать вектор $X^{[0, \ldots, T-1]}$. Аналогично через $X^{[\geqslant T]}, T<n$ будем обозначать вектор $X^{[T, \ldots, n-1]}$.

Далее будем пользоваться следующим взаимнооднозначным соответствием между двоичными векторами и целыми неотрицательными числами. Каждому целому неотрицательному числу соответствует двоичный вектор, в который данное число в двоичной системе счисления записано поразрядно. Аналогично, вектору $X=X_{n-1}\|\ldots\| X_{0}$ соответствует число $X_{n-1} \cdot 2^{n-1}+\ldots+X_{0} \cdot 2^{0}$. Тогда рассматривая числа, как двоичные векторы, можно к произвольному целому неотрицательному числу применять те же операции выделения подвекторов. 
Определение 2. Переносом в сумме $A+B+\ldots$ из младших $T$ бит в старшие называется число

$$
\sigma(A, B, \ldots ; t)=\left\lfloor\frac{A^{[<t]}+B^{[<t]}+\ldots}{2^{t}}\right\rfloor
$$

Младшие $T$ и старшие, начиная с $T$, бит суммы $A+B+\ldots$ можно записать следующим образом

$$
\begin{aligned}
& (A+B+\ldots)^{[<t]}=A^{[<t]} \boxplus B^{[<t]} \boxplus \ldots ; \\
& (A+B+\ldots)^{[\geqslant t]}=A^{[\geqslant t]}+B^{[\geqslant t]}+\ldots+\sigma(A, B, \ldots ; t) .
\end{aligned}
$$

Имеют место следующие факты, связанные с переносами, которые потребуются в дальнейшем. Через $a_{2}$ в дальнейшем будем обозначать представление числа $a$ в двоичной системе счисления.

Лемма 1. $B$ сумме $A+B+\alpha$, где $|A|=T,|B|=T, \alpha \in\left\{0_{2}, 1_{2}, 10_{2}\right\}$, перенос из младших $T$ разрядов в старшие не превосходит $10_{2} \equiv 2$.

Доказательство. $A \leqslant 2^{T}-1, B \leqslant 2^{T}-1 \Rightarrow A+B \leqslant 2^{T+1}-2=1 \underbrace{1 \ldots 10}_{T} 2$. Тогда $A+B+\alpha \leqslant 2^{T+1}=10 \underbrace{0 \ldots 0_{2}}_{T}$, то есть перенос не превосходит величины $10_{2}$. Лемма доказана.

Лемма 2. $B$ сумме $A+B+C+\alpha$, где $|A|=T,|B|=T,|C|=T, \alpha \in\left\{0_{2}, 1_{2}, 10_{2}\right\}$, перенос из младиих $T$ разрядов в старшие не превосходит $10_{2}$.

Доказательство. $A, B, C \leqslant 2^{T}-1 \Rightarrow A+B+C \leqslant 3 \cdot 2^{T}-3=10 \underbrace{1 \ldots 101_{2}}_{T}$. Тогда $A+B+C+\alpha \leqslant 10 \underbrace{1 \ldots 111}_{T} 2$, то есть перенос не превосходит величины $10_{2}$. Лемма доказана.

Лемма 3. ([2]) В сумме $A+B+\alpha$, где $|A|=T,|B|=T, \alpha \in\left\{0_{2}, 1_{2}\right\}$, перенос из младших $T$ разрядов в старшие не превосходит $1_{2}$.

Доказательство. Данная лемма и лемма 5 доказаны в расширенном варианте тезисов [2].

Имеем:

$$
A^{[<T]}+B^{[<T]}+\alpha<2^{T}+2^{T}-1+1=2 \cdot 2^{T} \Rightarrow\left\lfloor\frac{A^{[<T]}+B^{[<T]}+\alpha}{2^{T}}\right\rfloor \leqslant 1 .
$$

Лемма 4. Для любых $A, B, C, D,|A|=T,|B|=T,|C|=T,|D|=T$, верно равенство

$$
\sigma(A, B, C, D ; T)=\sigma(A, B, C ; T)+\sigma((A \boxplus B \boxplus C), D ; T) .
$$

Доказательство. По определению переноса имеем:

$$
A+B+C=\left\lfloor\frac{A+B+C}{2^{T}}\right\rfloor \cdot 2^{T}+(A \boxplus B \boxplus C),
$$




$$
\begin{aligned}
& A+B+C+D=\left\lfloor\frac{A+B+C}{2^{T}}\right\rfloor \cdot 2^{T}+(A \boxplus B \boxplus C)+D= \\
&=\left\lfloor\frac{A+B+C}{2^{T}}\right\rfloor \cdot 2^{T}+\left\lfloor\frac{(A \boxplus B \boxplus C)+D}{2^{T}}\right\rfloor \cdot 2^{T}+(A \boxplus B \boxplus C \boxplus D)= \\
&=\left\lfloor\frac{A+B+C}{2^{T}}\right\rfloor \cdot 2^{T}+\left\lfloor\frac{(A \boxplus B \boxplus C)+D}{2^{T}}\right\rfloor \cdot 2^{T}+(A \boxplus B \boxplus C \boxplus D)= \\
& \quad=\sigma(A, B, C ; T) \cdot 2^{T}+\sigma(A \boxplus B \boxplus C, D ; T) \cdot 2^{T}+(A \boxplus B \boxplus C \boxplus D) .
\end{aligned}
$$

Тогда

$$
\begin{aligned}
\sigma(A, B, C, D ; T)=\frac{A+B+C+D-(A \boxplus B \boxplus C \boxplus D)}{2^{T}} & = \\
& =\sigma(A, B, C ; T)+\sigma(A \boxplus B \boxplus C, D ; T) .
\end{aligned}
$$

Лемма 5. ([2]) Пусть $C_{1}, \ldots, C_{k}, k \in \mathbb{N},-$ произвольные целые неотрицательные числа, $T$ - натуральное число. Обозначим через $\gamma=\sigma\left(C_{1}, \ldots, C_{k} ; T\right)$ перенос из младших $T$ бит в старшие в сумме $C_{1}+\ldots+C_{k}$. Тогда для любого натурального $T$ имеет место равенство

$$
\sigma\left(C_{1}, \ldots, C_{k} ; T+t\right)=\sigma\left(C_{1}^{[\geqslant T]}, \ldots, C_{k}^{[\geqslant T]}, \gamma^{[<t]} ; t\right)+\gamma^{[\geqslant t]} .
$$

Доказательство. По определению

$$
\sigma\left(C_{1}, \ldots, C_{k} ; T+t\right)=\left\lfloor\frac{C_{1}^{[<T+t]}+\ldots+C_{k}^{[<T+t]}}{2^{T+t}}\right\rfloor .
$$

Используя представление (3), правую часть этого равенства запишем следующим образом:

$$
\begin{gathered}
\left\lfloor\frac{C_{1}^{[<T+t]}+\ldots+C_{k}^{[<T+t]}}{2^{T+t}}\right\rfloor= \\
=\left\lfloor\frac{C_{1}^{[<T]} \boxplus \ldots \boxplus C_{k}^{[<T]}}{2^{T+t}}+\frac{C_{1}^{[T, \ldots, T+t-1]}+\ldots+C_{k}^{[T, \ldots, T+t-1]}+\gamma}{2^{t}}\right\rfloor .
\end{gathered}
$$

Поскольку $\quad\left(C_{1}^{[<T]} \boxplus \ldots \boxplus C_{k}^{[<T]}\right) / 2^{T+t} \quad<\quad 1 / 2^{t}$, $\left(C_{1}^{[T, \ldots, T+t-1]}+\ldots+C_{k}^{[T, \ldots, T+t-1]}+\gamma\right) / 2^{t}$ имеет вид $N / 2^{t}$, где $N \in \mathbb{N} \cup\{0\}$, а для любых целых $N$ и и вещественного $0<L<1$ верно

$$
\left\lfloor\frac{N+L}{2^{t}}\right\rfloor=\left\lfloor\frac{N}{2^{t}}\right\rfloor
$$

то

$$
\begin{gathered}
\left\lfloor\frac{C_{1}^{[<T]} \boxplus \ldots \boxplus C_{k}^{[<T]}}{2^{T+t}}+\frac{C_{1}^{[T, \ldots, T+t-1]}+\ldots+C_{k}^{[T, \ldots, T+t-1]}+\gamma}{2^{t}}\right\rfloor= \\
=\left\lfloor\frac{C_{1}^{[T, \ldots, T+t-1]}+\ldots+C_{k}^{[T, \ldots, T+t-1]}+\gamma}{2^{t}}\right\rfloor .
\end{gathered}
$$


Подставляя в правую часть последнего равенства $\gamma=\gamma^{[<t]}+2^{t} \cdot \gamma^{[\geqslant t]}$, получим

$$
\begin{gathered}
\left\lfloor\frac{C_{1}^{[T, \ldots, T+t-1]}+\ldots+C_{k}^{[T, \ldots, T+t-1]}+\gamma}{2^{t}}\right\rfloor= \\
=\left\lfloor\frac{C_{1}^{[T, \ldots, T+t-1]}+\ldots+C_{k}^{[T, \ldots, T+t-1]}+\gamma^{[<t]}}{2^{t}}\right\rfloor+\gamma^{[\geqslant t]},
\end{gathered}
$$

что и требовалось доказать.

\section{4. Исследование разностного уравнения хэш-функции MD5}

Итак, требуется вычислить вероятность угадывания решения уравнения

$$
\begin{aligned}
\left(f\left(X_{1} \boxplus A_{1}, X_{2} \boxplus A_{2}, X_{3} \boxplus A_{3}\right) \boxplus X_{4} \boxplus A_{4}\right) & <<<s= \\
& =A_{0}<<<s \boxplus\left(f\left(X_{1}, X_{2}, X_{3}\right) \boxplus X_{4}\right)<<<s,
\end{aligned}
$$

при случайном и равномерном выборе значений переменных $X_{1}, \ldots, X_{4}$.

\section{1. Сведение разностного уравнения для MD5 к системе разностных} уравнений без сдвигов Из равенства векторов следует равенство подвекторов, поэтому уравнение (2) эквивалентно следующей системе (через $X|| Y$ обозначим конкатенацию векторов $X$ и $Y)$ :

$$
\left\{\begin{array}{l}
\left.f\left(X_{1} \boxplus A_{1}, X_{2} \boxplus A_{2}, X_{3} \boxplus A_{3}\right) \boxplus X_{4} \boxplus A_{4}\right)<<<s=B|| C,|B|=T-s,|C|=s, \\
\left.A_{0}<<<s \boxplus\left(f\left(X_{1}, X_{2}, X_{3}\right) \boxplus X_{4}\right)<<<s\right)=D \| E,|D|=T-s,|E|=s, \\
B=D, \\
C=E .
\end{array}\right.
$$

Очевидно, что

$$
f\left(X_{1} \boxplus A_{1}, X_{2} \boxplus A_{2}, X_{3} \boxplus A_{3}\right) \boxplus X_{4} \boxplus A_{4}=C|| B .
$$

Выпишем выражение для $B$ :

$$
\begin{aligned}
B=f\left(X_{1}^{[<T-s]} \boxplus A_{1}^{[<T-s]}, X_{2}^{[<T-s]} \boxplus A_{2}^{[<T-s]}, X_{3}^{[<T-s]} \boxplus A_{3}^{[<T-s]}\right) \boxplus & \boxplus X_{4}^{[<T-s]} \boxplus A_{4}^{[<T-s]} .
\end{aligned}
$$

Введем обозначения для переносов:

- $\sigma_{1}$ - перенос из младших T-s бит в сумме $X_{1}+A_{1} ; \sigma_{1} \leqslant 1$, согласно лемме 3 .

- $\sigma_{2}$ - перенос из младших T-s бит в сумме $X_{2}+A_{2} ; \sigma_{2} \leqslant 1$, согласно лемме 3 .

- $\sigma_{3}$ - перенос из младших T-s бит в сумме $X_{3}+A_{3} ; \sigma_{3} \leqslant 1$, согласно лемме 3 .

- $\sigma_{4}$ - перенос из младших Т-s бит в сумме $f\left(X_{1}+A_{1}, X_{2}+A_{2}, X_{3}+A_{3}\right)+X_{4}+A_{4}$;

$\sigma_{4} \leqslant 2$, согласно лемме 2 .

Выпишем выражение для $C$ :

$C=f\left(X_{1}^{[\geqslant T-s]} \boxplus A_{1}^{[\geqslant T-s]} \boxplus \sigma_{1}, X_{2}^{[\geqslant T-s]} \boxplus A_{2}^{[\geqslant T-s]} \boxplus \sigma_{2}, X_{3}^{[\geqslant T-s]} \boxplus A_{3}^{[\geqslant T-s]} \boxplus \sigma_{3}\right) \boxplus$ $\boxplus X_{4}^{[\geqslant T-s]} \boxplus A_{4}^{[\geqslant T-s]} \boxplus \sigma_{4}$. 
Выпишем выражения для $D$ и $E$.

Для простоты разобьем слагаемые из левой части на подвекторы длины $s$ и $T-s$.

$$
\begin{gathered}
A_{0}<<<s=P_{1}|| P_{0},\left|P_{0}\right|=s,\left|P_{1}\right|=T-s ; \\
\left(f\left(X_{1}, X_{2}, X_{3}\right) \boxplus X_{4}\right)<<<s=Q_{1}|| Q_{0},\left|Q_{0}\right|=s,\left|Q_{1}\right|=T-s .
\end{gathered}
$$

Введем обозначения для переносов:

- $\sigma_{0}$ - перенос из младших $s$ бит в сумме $P_{0}+Q_{0}$.

- $\sigma_{5}$ - перенос из младших $T-s$ бит в сумме $f\left(X_{1}, X_{2}, X_{3}\right)+X_{4} ; \sigma_{5} \leqslant 1$, согласно лемме 3 .

Тогда

$$
\begin{gathered}
E=P_{0} \boxplus Q_{0}, \\
D=P_{1} \boxplus Q_{1} \boxplus \sigma_{0} .
\end{gathered}
$$

Далее,

$$
\begin{gathered}
P_{1}=A_{0}^{[<T-s]}, P_{0}=A_{0}^{[\geqslant T-s]}, \\
Q_{1}=f\left(X_{1}^{[<T-s]}, X_{2}^{[<T-s]}, X_{3}^{[<T-s]}\right) \boxplus X_{4}^{[<T-s]}, \\
Q_{0}=f\left(X_{1}^{[\geqslant T-s]}, X_{2}^{[\geqslant T-s]}, X_{3}^{[\geqslant T-s]}\right) \boxplus X_{4}^{[\geqslant T-s]} \boxplus \sigma_{5}, \\
\sigma_{0}=\left(\sigma\left(A_{0}^{[\geqslant T-s]}, f\left(X_{1}^{[\geqslant T-s]}, X_{2}^{[\geqslant T-s]}, X_{3}^{[\geqslant T-s]}\right) \boxplus X_{4}^{[\geqslant T-s]} \boxplus \sigma_{5} ; s\right) .\right.
\end{gathered}
$$

Согласно лемме 3 перенос $\sigma_{0} \leqslant 1$.

Итак,

$$
\begin{gathered}
E=A_{0}^{[\geqslant T-s]} \boxplus f\left(X_{1}^{[\geqslant T-s]}, X_{2}^{[\geqslant T-s]}, X_{3}^{[\geqslant T-s]}\right) \boxplus X_{4}^{[\geqslant T-s]} \boxplus \sigma_{5} \\
D=A_{0}^{[<T-s]} \boxplus f\left(X_{1}^{[<T-s]}, X_{2}^{[<T-s]}, X_{3}^{[<T-s]}\right) \boxplus X_{4}^{[<T-s]} \boxplus \sigma_{0} .
\end{gathered}
$$

В результате можно выписать систему уравнений, эквивалентную уравнению (2):

$$
\left\{\begin{array}{c}
f\left(X_{1}^{[<T-s]} \boxplus A_{1}^{[<T-s]}, X_{2}^{[<T-s]} \boxplus A_{2}^{[<T-s]}, X_{3}^{[<T-s]} \boxplus A_{3}^{[<T-s]}\right) \boxplus A_{4}^{[<T-s]}= \\
=A_{0}^{[<T-s]} \boxplus f\left(X_{1}^{[<T-s]}, X_{2}^{[<T-s]}, X_{3}^{[<T-s]}\right) \boxplus \sigma_{0}, \\
f\left(X_{1}^{[\geqslant T-s]} \boxplus A_{1}^{[\geqslant T-s]} \boxplus \sigma_{1}, X_{2}^{[\geqslant T-s]} \boxplus A_{2}^{[\geqslant T-s]} \boxplus \sigma_{2}, X_{3}^{[\geqslant T-s]} \boxplus A_{3}^{[\geqslant T-s]} \boxplus \sigma_{3}\right) \boxplus \\
\boxplus A_{4}^{[\geqslant T-s]} \boxplus \sigma_{4}=A_{0}^{[\geqslant T-s]} \boxplus f\left(X_{1}^{[\geqslant T-s]}, X_{2}^{[\geqslant T-s]}, X_{3}^{[\geqslant T-s]}\right) \boxplus \sigma_{5} .
\end{array}\right.
$$

\section{5. Анализ разностного уравнения с переносами более общего вида}

В данном разделе рассмотрим уравнение вида

$$
f\left(X_{1} \boxplus A_{1}, X_{2} \boxplus A_{2}, X_{3} \boxplus A_{3}\right) \boxplus A_{4} \boxplus X_{4}=f\left(X_{1}, X_{2}, X_{3}\right) \boxplus X_{4} \boxplus A_{0},
$$

где $f$ - булева функция трех переменных, применяемая к аргументам побитово, $\left|X_{i}\right|=T, i=\overline{1,4},\left|A_{i}\right|=T, i=\overline{0,4}$. В следующем разделе будет показано, что анализ каждого из уравнений системы (6) можно свести к анализу данного уравнения. 
Введем ряд обозначений. Совокупность уравнения (7) и выражения относительно тех же переменных $f\left(X_{1}, X_{2}, X_{3}\right) \boxplus X_{4}$ обозначим через $\mathcal{E}(f, T, A)$. Далее, пусть $\alpha=\left(\alpha_{0}, \ldots, \alpha_{5}\right) \in\left\{0_{2}, 1_{2}, 10_{2}\right\}^{6}$, тогда через $\mathcal{E}_{\alpha}(f, T, A)$ обозначим совокупность $\mathcal{E}(f, T, A)$, в уравнении которой к каждому из коэффициентов $A_{i}$ прибавлена по модулю $2^{T}$ величина $\alpha_{i}$, а к выражению $f\left(X_{1}, X_{2}, X_{3}\right) \boxplus X_{4}$ прибавлена по модулю $2^{T}$ величина $\alpha_{5}$.

Введем обозначения для следующих переносов из младших разрядов в старшие:

- $\gamma_{1}, \gamma_{2}, \gamma_{3}$ - переносы из младших $T$ бит в суммах $X_{1}+A_{1}+\alpha_{1}, X_{2}+A_{2}+\alpha_{2}$, $X_{3}+A_{3}+\alpha_{3}$ (данные переносы не превосходят $2-$ см. лемму 1 ),

- $\gamma_{4}$ - перенос из младших $T$ бит в сумме $f\left(X_{1} \boxplus A_{1} \boxplus \alpha_{1}, X_{2} \boxplus A_{2} \boxplus \alpha_{2}, X_{3} \boxplus\right.$ $\left.A_{3} \boxplus \alpha_{3}\right)+X_{4}+A_{4}+\alpha_{4}$ (данный перенос не превосходит $2-$ см. лемму 2 ),

- $\gamma_{0}$ - перенос из младших $T$ бит в сумме $A_{0}+f\left(X_{1}, X_{2}, X_{3}\right)+X_{4}+\alpha_{0}$ (данный перенос не превосходит $2-$ см. лемму 2$)$.

- $\gamma_{5}$ - перенос из младших $T$ бит в сумме $f\left(X_{1}, X_{2}, X_{3}\right)+X_{4}+\alpha_{5}$ (данный перенос не превосходит $2-$ см. лемму 2).

Совокупность величин $\gamma_{0}, \ldots, \gamma_{5}$ будем называть вектором переносов.

Через $P(f, T, A)$ обозначим вероятность угадывания решения уравнения из $\mathcal{E}(f, T, A)$, при равновероятном и независимом выборе значений неизвестных $X$, в данном случае на значение выражения $f\left(X_{1}, X_{2}, X_{3}\right) \boxplus X_{4}$ ограничений не накладывается. Через $P_{\alpha}(f, T, A)$, где $\alpha \in\left(\left\{0_{2}, 1_{2}, 10_{2}\right\}\right)^{6}$, обозначим вероятность угадывания решения уравнения из $\mathcal{E}\left(f, T, A \boxplus \alpha^{[0,1,2,3,4]}\right)$ (сложение векторов осуществляется покомпонентно), при этом к выражению $f\left(X_{1}, X_{2}, X_{3}\right) \boxplus X_{4}$ прибавляется $\alpha_{5}$. Через $P^{\beta}(f, T, A)$ обозначим вероятность угадывания решения уравнения из $\mathcal{E}(f, T, A)$ такого, что перечисленные выше в данном разделе переносы были равны вектору $\beta=\left(\beta_{0}, \ldots, \beta_{5}\right) \in\left\{0_{2}, 1_{2}, 10_{2}\right\}^{6}$. Соответственно через $P_{\alpha}^{\beta}(f, T, A)$ обозначим вероятность угадывания решения уравнения из $\mathcal{E}_{\alpha}(f, T, A)$ такого, что соответствующий ему вектор переносов был равен вектору $\beta$. Через $\mathcal{E}_{\alpha}^{\beta}(f, T, A)$ обозначим $\mathcal{E}_{\alpha}(f, T, A)$, для решения уравнения из которого необходимо равенство значению $\beta$ вектора переносов $\gamma$. Через $\mathcal{E}^{\beta}(f, T, A)$ обозначим $\mathcal{E}(f, T, A)$, для решения уравнения из которого необходимо равенство значению $\beta$ вектора переносов $\gamma$.

Теорема 1. Набор $X^{[<T+t]}$ (при $\left.T, t \geqslant 2\right)$ является решением уравнения из $\mathcal{E}_{\alpha}(f, T+t, A)$, где $A \in\left(\{0,1\}^{t+T}\right)^{5}, \alpha \in\left\{0_{2}, 1_{2}, 10_{2}\right\}^{6}$, с вектором переносов $\gamma \in\left\{0_{2}, 1_{2}, 10_{2}\right\}^{6}$, тогда и только тогда, когда:

(1) набор $X^{[<T]}$ является решением уравнения из $\mathcal{E}_{\alpha}\left(f, T, A^{[<T]}\right)$ с некоторым вектором переносов $\sigma=\left(\sigma_{0}, \sigma_{1}, \sigma_{2}, \sigma_{3}, \sigma_{4}, \sigma_{5}\right)$ в соответствующих суммах из младиих T бит в стариие;

(2) набор $X^{[T, \ldots, T+t-1]}$ является решением уравнения из $\mathcal{E}_{\sigma}\left(f, t, A^{[\geqslant T]}\right)$ с вектором переносов $\gamma$ в соответствующих суммах из младиих $T$ бит в стариие.

Доказательство. Рассмотрим левую и правую части уравнения как векторы и разобьем их на два подвектора с длинами $T$ и $t$ соответственно. Тогда уравнение можно переписать в виде системы, так как равенство векторов подразумевает и равенство подвекторов с совпадающими начальным и конечным индексами:

$$
f\left(X_{1} \boxplus A_{1} \boxplus \alpha_{1}, X_{2} \boxplus A_{2} \boxplus \alpha_{2}, X_{3} \boxplus A_{3} \boxplus \alpha_{3}\right) \boxplus A_{4} \boxplus X_{4} \boxplus \alpha_{4}=f\left(X_{1}, X_{2}, X_{3}\right) \boxplus X_{4} \boxplus A_{0} \boxplus \alpha_{0}
$$




$$
\left\{\begin{array}{r}
\left(f\left(X_{1} \boxplus A_{1} \boxplus \alpha_{1}, X_{2} \boxplus A_{2} \boxplus \alpha_{2}, X_{3} \boxplus A_{3} \boxplus \alpha_{3}\right) \boxplus A_{4} \boxplus X_{4} \boxplus \alpha_{4}\right)^{[<T]}= \\
=\left(f\left(X_{1}, X_{2}, X_{3}\right) \boxplus X_{4} \boxplus A_{0} \boxplus \alpha_{0}\right)^{[<T]}, \\
\left(f\left(X_{1} \boxplus A_{1} \boxplus \alpha_{1}, X_{2} \boxplus A_{2} \boxplus \alpha_{2}, X_{3} \boxplus A_{3} \boxplus \alpha_{3}\right) \boxplus X_{4} \boxplus A_{4} \boxplus \alpha_{4}\right)^{[T, \ldots, T+t-1]}= \\
=\left(f\left(X_{1}, X_{2}, X_{3}\right) \boxplus X_{4} \boxplus A_{0} \boxplus \alpha_{0}\right)^{[T, \ldots, T+t-1]}
\end{array}\right.
$$

Далее воспользуемся соотношениями для переносов из раздела 3. Последняя система эквивалентна системе

$$
\left\{\begin{array}{r}
f\left(\left(X_{1} \boxplus A_{1} \boxplus \alpha_{1}\right)^{[<T]},\left(X_{2} \boxplus A_{2} \boxplus \alpha_{2}\right)^{[<T]},\left(X_{3} \boxplus A_{3} \boxplus \alpha_{3}\right)^{[<T]}\right) \boxplus A_{4}^{[<T]} \boxplus X_{4}^{[<T]} \boxplus \\
\boxplus \alpha_{4}=f\left(X_{1}^{[<T]}, X_{2}^{[<T]}, X_{3}^{[<T]}\right) \boxplus X_{4}^{[<T]} \boxplus A_{0}^{[<T]} \boxplus \alpha_{0}, \\
f\left(\left(X_{1} \boxplus A_{1} \boxplus \alpha_{1}\right)^{[T, \ldots, T+t-1]}, \ldots,\left(X_{3} \boxplus A_{3} \boxplus \alpha_{3}\right)^{[T, \ldots, T+t-1]}\right) \boxplus X_{4}^{[T, \ldots, T+t-1]} \boxplus \\
\boxplus A_{4}^{[T, \ldots, T+t-1]} \boxplus \sigma_{4}=f\left(X_{1}^{[T, \ldots, T+t-1]}, \ldots, X_{3}^{[T, \ldots, T+t-1]}\right)^{[T, \ldots, T+t-1]} \boxplus \\
X_{4}^{[T, \ldots, \ldots, T+t-1]} \boxplus \sigma_{0} .
\end{array}\right.
$$

А она, учитывая равенство $\alpha_{i}^{[T, \ldots, T+t-1]}=0$, эквивалентна системе

$$
\left\{\begin{array}{r}
f\left(X_{1}^{[<T]} \boxplus A_{1}^{[<T]} \boxplus \alpha_{1}, \ldots, X_{3}^{[<T]} \boxplus A_{3}^{[<T]} \boxplus \alpha_{3}\right) \boxplus A_{4}^{[<T]} \boxplus X_{4}^{[<T]} \boxplus \alpha_{4}= \\
=f\left(X_{1}^{[<T]}, X_{2}^{[<T]}, X_{3}^{[<T]}\right) \boxplus X_{4}^{[<T]} \boxplus A_{0}^{[<T]} \boxplus \alpha_{0}, \\
f\left(X_{1}^{[T, \ldots, T+t-1]} \boxplus A_{1}^{[T, \ldots, T+t-1]} \boxplus \sigma_{1}, \ldots X_{3}^{[T, \ldots, T+t-1]} \boxplus A_{3}^{[T, \ldots, T+t-1]} \boxplus \sigma_{3}\right) \boxplus \\
\boxplus A_{4}^{[T, \ldots, T+t-1]} \boxplus \sigma_{4}=f\left(X_{1}^{[T, \ldots, T+t-1]}, X_{2}^{[T, \ldots, T+t-1]}, X_{3}^{[T, \ldots, T+t-1]}\right) \boxplus \\
\boxplus A_{0}^{[T, \ldots, T+t-1]} \boxplus \sigma_{0} .
\end{array}\right.
$$

Таким образом, установлен тот факт, что решение уравнения $\mathcal{E}_{\alpha}(f, T+t, A)$ можно представить в виде конкатенации решений уравнений из $\mathcal{E}_{\sigma}\left(f, t, A^{[T, \ldots, T+t-1]}\right)$ и $\mathcal{E}_{\alpha}^{\sigma}\left(f, T, A^{[<T]}\right)$.

Точно так же показывается выполнение системы равенств

$$
\left\{\begin{array}{l}
\left(f\left(X_{1}, X_{2}, X_{3}\right) \boxplus X_{4} \boxplus \alpha_{5}\right)^{[<T]}=f\left(X_{1}^{[<T]}, X_{2}^{[<T]}, X_{3}^{[<T]}\right) \boxplus X_{4}^{[<T]} \boxplus \alpha_{5} \\
\left(f\left(X_{1}, X_{2}, X_{3}\right) \boxplus X_{4} \boxplus \alpha_{5}\right)^{[T, \ldots, T+t-1]}= \\
\quad=f\left(X_{1}^{[T, \ldots, T+t-1]}, X_{2}^{[T, \ldots, T+t-1]}, X_{3}^{[T, \ldots, T+t-1]}\right) \boxplus X_{4}^{[T, \ldots, T+t-1]} \boxplus \sigma_{5}
\end{array}\right.
$$

Совокупность систем (8) и (9) эквивалентна записи

$$
\left\{\begin{array}{l}
\mathcal{E}_{\alpha}^{\sigma}\left(f, T, A^{[<T]}\right) \\
\mathcal{E}_{\sigma}\left(f, t, A^{[T, \ldots, T+t-1]}\right) .
\end{array}\right.
$$

Осталось показать, что для набора $X^{[<T+t]}$ и уравнения из $\mathcal{E}_{\alpha}(f, T+t, A)$ вектор переносов из младших $T+t$ бит в старшие в соответствующих суммах совпадает с вектором переносов из младших $T$ бит в старшие для набора $X^{[T, \ldots, T+t-1]}$ и уравнения из $\mathcal{E}_{\sigma}\left(f, t, A^{[T, \ldots, T+t-1]}\right)$. 
Покажем, что это утверждение верно для $\gamma_{4}$. Для остальных переносов доказательство аналогично. Требуется показать, что

$$
\begin{aligned}
& \gamma_{4}=\sigma\left(f\left(X_{1} \boxplus A_{1} \boxplus \alpha_{1}, X_{2} \boxplus A_{2} \boxplus \alpha_{2}, X_{3} \boxplus A_{3} \boxplus \alpha_{3}\right), A_{4}, X_{4}, \alpha_{4} ; T+t\right)= \\
& =\sigma\left(f\left(X_{1}^{[T, \ldots, T+t-1]} \boxplus A_{1}^{[T, \ldots, T+t-1]} \boxplus \sigma_{1}, \ldots, X_{3}^{[T, \ldots, T+t-1]} \boxplus A_{3}^{[T, \ldots, T+t-1]} \boxplus \sigma_{3}\right),\right. \\
& \left.A_{4}^{[T, \ldots, T+t-1]}, X_{4}^{[T, \ldots, T+t-1]}, \sigma_{4} ; t\right) .
\end{aligned}
$$

Так как $Y^{[T, \ldots, T+t-1]}=Y^{\geqslant T}$, где $Y$ - любое из слагаемых суммы $f\left(X_{1} \boxplus A_{1} \boxplus\right.$ $\left.\alpha_{1}, X_{2} \boxplus A_{2} \boxplus \alpha_{2}, X_{3} \boxplus A_{3} \boxplus \alpha_{3}\right) \boxplus A_{4} \boxplus X_{4} \boxplus \alpha_{4}$, то согласно лемме 5 имеем

$$
\begin{aligned}
& \sigma\left(f\left(X_{1} \boxplus A_{1} \boxplus \alpha_{1}, \ldots\right), A_{4}, X_{4}, \alpha_{4} ; T+t\right)= \\
= & \sigma\left(f\left(X_{1} \boxplus A_{1} \boxplus \alpha_{1}, \ldots\right)^{[T, \ldots, T+t-1]}, A_{4}^{[T, \ldots, T+t-1]}, \alpha_{4}^{[T, \ldots, T+t-1]}, X_{4}^{[T, \ldots, T+t-1]}, \sigma_{4}^{[<t]} ; t\right)+ \\
& +\sigma_{4}^{[T, \ldots, T+t-1]} .
\end{aligned}
$$

Но, так как $\alpha_{j}, \sigma_{j} \in\left\{0_{2}, 1_{2}, 10_{2}\right\}$ ( $\alpha$ - согласно условию теоремы, $\sigma-$ согласно леммам раздела 3$)$, а, следовательно, $\alpha_{j}^{[\geqslant 2]}=\sigma^{[\geqslant 2]}=0$, то

$$
\begin{gathered}
\sigma\left(f\left(X_{1} \boxplus A_{1} \boxplus \alpha_{1}, \ldots, X_{3} \boxplus A_{3} \boxplus \alpha_{3}\right)^{[T, \ldots, T+t-1]}, X_{4}^{[T, \ldots, T+t-1]}, A_{4}^{[T, \ldots, T+t-1]},\right. \\
\left.\alpha_{4}^{[T, \ldots, T+t-1]}, \sigma_{4}^{[<t]} ; t\right)+\sigma_{4}^{[T, \ldots, T+t-1]}= \\
=\sigma\left(f\left(\left(X_{1} \boxplus A_{1} \boxplus \alpha_{1}\right)^{[T, \ldots, T+t-1]}, \ldots, X_{3} \boxplus A_{3} \boxplus \alpha_{3}\right)^{[T, \ldots, T+t-1]}\right), \\
\left.X_{4}^{[T, \ldots, T+t-1]}, A_{4}^{[T, \ldots, T+t-1]}, \sigma_{4} ; t\right)= \\
=\sigma\left(f \left(X_{1}^{[T, \ldots, T+t-1]} \boxplus A_{1}^{[T, \ldots, T+t-1]} \boxplus \alpha_{1}^{[T, \ldots, T+t-1]} \boxplus \sigma_{1}, \ldots, X_{3}^{[T, \ldots, T+t-1]} \boxplus\right.\right. \\
\left.\left.\boxplus A_{3}^{[T, \ldots, T+t-1]} \boxplus \alpha_{3}^{[T, \ldots, T+t-1]} \boxplus \sigma_{3}\right), X_{4}^{[T, \ldots, T+t-1]}, A_{4}^{[T, \ldots, T+t-1]}, \sigma_{4} ; t\right)= \\
=\sigma\left(f\left(X_{1}^{[T, \ldots, T+t-1]} \boxplus A_{1}^{[T, \ldots, T+t-1]} \boxplus \sigma_{1}, \ldots, X_{3}^{[T, \ldots, T+t-1]} \boxplus A_{3}^{[T, \ldots, T+t-1]} \boxplus \sigma_{3}\right),\right. \\
\left.X_{4}^{[T, \ldots, T+t-1]}, A_{4}^{[T, \ldots, T+t-1]}, \sigma_{4} ; t\right) .
\end{gathered}
$$

Итак, соотношение для $\gamma_{4}$ установлено.

Теорема доказана.

Воспользуемся еще одним обозначением из работы [2]. Через $\left|\mathcal{E}_{\alpha}^{\beta}(f, T, A)\right|$ обозначается количество таких решений уравнения $\mathcal{E}_{\alpha}(f, T, A)$, для которых вектор переносов равен вектору $\beta$. Поскольку вектор $X$ выбирается случайно и равновероятно, вероятность угадывания решения уравнения $\mathcal{E}_{\alpha}(f, T, A)$ с заданными переносами будет равна

$$
P_{\alpha}^{\beta}(f, T, A)=\frac{\left|\mathcal{E}_{\alpha}^{\beta}(f, T, A)\right|}{2^{4 T}} .
$$

Следствие 1. Для любых $A \in\left(\{0,1\}^{T+t}\right)^{5}, \alpha \in\left\{0_{2}, 1_{2}, 10_{2}\right\}^{6}, \beta \in\left\{0_{2}, 1_{2}, 10_{2}\right\}^{6}$, $T \geqslant 2, t \geqslant 2$ и произвольной булевой функции $f$ имеет место равенство

$$
\left|\mathcal{E}_{\alpha}^{\beta}(f, T+t, A)\right|=\sum_{\gamma \in\left\{0_{2}, 1_{2}, 10_{2}\right\}^{6}}\left|\mathcal{E}_{\gamma}^{\beta}\left(f, t, A^{[T, \ldots, T+t]}\right)\right| \cdot\left|\mathcal{E}_{\alpha}^{\gamma}\left(f, T, A^{[<T]}\right)\right| .
$$


Следствие 2. Для любых $A \in\left(\{0,1\}^{T+t}\right)^{5}, \alpha \in\left\{0_{2}, 1_{2}, 10_{2}\right\}^{6}, \beta \in\left\{0_{2}, 1_{2}, 10_{2}\right\}^{6}$, $T \geqslant 2, t \geqslant 2$ и произвольной булевой функиии $f$ имеет место равенство

$$
P_{\alpha}^{\beta}(f, T+t, A)=\sum_{\gamma \in\left\{0_{2}, 1_{2}, 10_{2}\right\}^{6}} P_{\gamma}^{\beta}\left(f, t, A^{[T, \ldots, T+t]}\right) \cdot P_{\alpha}^{\gamma}\left(f, T, A^{[<T]}\right) .
$$

Обозначим через $M(f, A, T)$ матрицу размера $3^{6} \times 3^{6}$. Для каждого элемента данной матрицы представление в троичной системе счисления номера его строки обозначим вектором $\alpha$, а представление в троичной системе счисления номера его столбца обозначим вектором $\beta$. В ячейках матрицы $M(f, A, T)$ находятся значения $P_{\alpha}^{\beta}(f, A, T)$. Эту матрицу будем называть вероятностной матрицей для уравнения вида (7).

5.1. Соотношения для вероятностных матриц Теперь установим ряд соотношений для вероятностных матриц, определенных выше.

Лемма 6. Для любых $A \in\left(\{0,1\}^{T+t}\right)^{5}, T, t \geqslant 2$ выполнено следующее рекуррентное соотношение:

$$
M(f, A, T+t)=M\left(f, A^{[T, \ldots, T+t-1]}, t\right) \cdot M\left(f, A^{[<T]}, T\right) .
$$

Доказательство. Фактически нужно доказать равенство

$$
P_{\alpha}^{\beta}(f, T+t, A)=\sum_{\gamma \in\left\{0_{2}, 1_{2}, 10_{2}\right\}^{6}} P_{\gamma}^{\beta}\left(f, t, A^{[T, \ldots, T+t-1]}\right) \cdot P_{\alpha}^{\gamma}\left(f, T, A^{[<T]}\right) .
$$

Оно верно в соответствии со следствием 2.

Лемма доказана.

Следствие 3. Для любого четного $T \geqslant 4$ верно равенство

$$
M(f, A, T)=\prod_{i=\frac{T}{2}-1}^{0} M\left(f, A^{[i \cdot 2, i \cdot 2+1]}, 2\right) .
$$

Следствие 4. Для любого нечетного $T \geqslant 5$ верно равенство

$$
M(f, A, T)=M\left(f, A^{[T-3, T-2, T-1]}, 3\right) \cdot \prod_{i=\frac{T-3}{2}-1}^{0} M\left(f, A^{[i \cdot 2, i \cdot 2+1]}, 2\right) .
$$

Отсюда, в частности, следует, что $M(f ; A ; T)=M\left(f ; A^{[<T]} ; T\right)$. Другими словами, матрица $M(f ; A ; T)$, а значит, и все функции от нее, зависят только от $T$ младших бит параметров $A_{i}$.

\section{6. Анализ системы разностных уравнений для MD5}

Вернемся к системе (6). Напомним, что она имела вид

$$
\left\{\begin{array}{c}
f\left(X_{1}^{[<T-s]} \boxplus A_{1}^{[<T-s]}, X_{2}^{[<T-s]} \boxplus A_{2}^{[<T-s]}, X_{3}^{[<T-s]} \boxplus A_{3}^{[<T-s]}\right) \boxplus A_{4}^{[<T-s]}= \\
=A_{0}^{[<T-s]} \boxplus f\left(X_{1}^{[<T-s]}, X_{2}^{[<T-s]}, X_{3}^{[<T-s]}\right) \boxplus \sigma_{0}, \\
f\left(X_{1}^{[\geqslant T-s]} \boxplus A_{1}^{[\geqslant T-s]} \boxplus \sigma_{1}, X_{2}^{[\geqslant T-s]} \boxplus A_{2}^{[\geqslant T-s]} \boxplus \sigma_{2}, X_{3}^{[\geqslant T-s]} \boxplus A_{3}^{[\geqslant T-s]} \boxplus \sigma_{3}\right) \boxplus \\
\boxplus A_{4}^{[\geqslant T-s]} \boxplus \sigma_{4}=A_{0}^{[\geqslant T-s]} \boxplus f\left(X_{1}^{[\geqslant T-s]}, X_{2}^{[\geqslant T-s]}, X_{3}^{[\geqslant T-s]}\right) \boxplus \sigma_{5} .
\end{array}\right.
$$


Зафиксируем произвольные значения для параметров $A_{0}, \ldots, A_{4}$.

Задача состояла в том, чтобы вычислить вероятность угадывания решения уравнения (2), случайно и равновероятно выбирая значения переменных $X_{0}, \ldots, X_{4}$. Ранее было показано, что вектор $X$ является решением уравнения (2) тогда и только тогда, когда выполнены следующие условия:

- подвектор $X^{[<T-s]}$ является решением уравнения из $\mathcal{E}^{\sigma}\left(f, T, A^{[<T]}\right)$ для некоторого вектора $\sigma$;

- подвектор $X^{[\geqslant T-s]}$ является решением уравнения из $\mathcal{E}_{\sigma}\left(f, t, A^{[T, \ldots, T+t-1]}\right)$.

6.1. Вычисление вероятности угадывания решения уравнения В этом разделе будет показано, как свести вычисление вероятности $P(f, T, A)$ к вычислению суммы диагональных элементов произведения вероятностных матриц.

Сначала покажем, что каждое из уравнений системы (6) представляет собой уравнение (7), а переносы $\sigma_{j}$ можно представить через переносы $\gamma_{i}$, описанные выше.

Лемма 7. Первое уравнение системы (6) является уравнением из $\mathcal{E}_{\left\{\alpha_{0}, 0,0,0,0,0\right\}}\left(f, A^{[<T-s]}, T-s\right) . \quad$ При этом переносы $\gamma_{1}, \ldots, \gamma_{5}$ из определения уравнения (7) в точности совпадают по определению с переносами $\sigma_{1}, \ldots, \sigma_{5}$ из системы (6), значения которых определяются младиими Т-s битами перемен$\operatorname{Habx} X_{i}$.

Доказательство. Действительно, уравнение из $\mathcal{E}_{\left\{\alpha_{0}, 0,0,0,0,0\right\}}\left(f, A^{[<T-s]}, T-s\right)$ и первое уравнение системы (6) совпадают. Также совпадают и векторы переносов $\sigma_{1}, \ldots, \sigma_{5}$ и $\gamma_{1}, \ldots, \gamma_{5}$.

Лемма доказана.

Лемма 8. Второе уравнение системы является уравнением из $\mathcal{E}_{\left(\sigma_{5}, \sigma_{1}, \sigma_{2}, \sigma_{3}, \sigma_{4}, \sigma_{5}\right)}\left(f, A^{[\geqslant T-s]}, T-s\right) . \quad$ При этом перенос $\sigma_{0}$, для которого требуется равенство заданной величине, равен $\gamma_{0}-\gamma_{5}$.

Доказательство. Из вида второго уравнения системы (6) следует выполнение первой части утверждения леммы - действительно, к каждой сумме $X_{i}+A_{i}, i=1,2,3$ прибавляется перенос $\sigma_{i}$, к левой части уравнения дополнительно прибавляется $\sigma_{4}$, к правой $-\sigma_{5}$.

Теперь осталось показать справедливость утверждения относительно переносов. В уравнении, рассмотренном в Теореме 1 , перенос $\gamma_{0}$ равен $\sigma\left(A_{0}, f\left(X_{1}, X_{2}, X_{3}\right), X_{4}, \alpha_{0} ; s\right), \quad$ перенос $\quad \gamma_{5} \quad$ равен $\quad \sigma\left(f\left(X_{1}, X_{2}, X_{3}\right), X_{4}, \alpha_{5} ; s\right)$ (по условию теоремы $\alpha_{5}=\alpha_{0}=\sigma_{5}$ ), a перенос $\sigma_{0}$ равен $\sigma\left(A_{0}^{[\geqslant T-s]}, f\left(X_{1}^{[\geqslant T-s]}, X_{2}^{[\geqslant T-s]}, X_{3}^{[\geqslant T-s]}\right) \boxplus X_{4}^{[\geqslant T-s]} \boxplus \sigma_{5} ; s\right)$. Согласно Лемме 4

$$
\begin{aligned}
\sigma\left(A_{0}^{[\geqslant T-s]}, f\left(X_{1}^{[\geqslant T-s]}, X_{2}^{[\geqslant T-s]}, X_{3}^{[\geqslant T-s]}\right), X_{4}^{[\geqslant T-s]}, \alpha_{0} ; s\right)= \\
=\sigma\left(f\left(X_{1}^{[\geqslant T-s]}, X_{2}^{[\geqslant T-s]}, X_{3}^{[\geqslant T-s]}\right), X_{4}^{[\geqslant T-s]}, \alpha_{0} ; s\right)+ \\
\quad+\sigma\left(A_{0}^{[\geqslant T-s]}, f\left(X_{1}^{[\geqslant T-s]}, X_{2}^{[\geqslant T-s]}, X_{3}^{[\geqslant T-s]}\right) \boxplus X_{4}^{[\geqslant T-s]} \boxplus \alpha_{0} ; s\right) .
\end{aligned}
$$

Лемма доказана.

Вероятность угадывания решения первого уравнения системы в точности совпадает с вероятностью угадать решение уравнения из Теоремы 1 , а вероятность 
угадывания решения второго отличается переносом $\sigma_{0}$. Данный перенос отличается от исследованного в уравнении (7) на $-\gamma_{5}$.

Введем несколько обозначений. Через $R_{1}^{\sigma_{1}, \ldots, \sigma_{5}}\left(\sigma_{0}\right)$ обозначим вероятность угадывания решения первого уравнения системы так, что для решения соответствующие переносы будут равны $\sigma_{1}, \ldots, \sigma_{5}$. Аналогично через $R_{2}^{\sigma_{0}}\left(\sigma_{1}, \ldots, \sigma_{5}\right)$ обозначим вероятность угадывания такого решения второго уравнения системы, чтобы соответствующий перенос был равен $\sigma_{0}$.

Тогда вероятность угадывания решения системы равна

$$
P(f, T, A)=\sum_{\sigma_{0}, \ldots, \sigma_{5} \in\left\{0_{2}, 1_{2}, 10_{2}\right\}} R_{1}^{\sigma_{1}, \ldots, \sigma_{5}}\left(\sigma_{0}\right) \cdot R_{2}^{\sigma_{0}}\left(\sigma_{1}, \ldots, \sigma_{5}\right)
$$

Рассмотрим первое уравнение системы (6). Необходимо получить такое решение, чтобы переносы $\sigma_{1}, \sigma_{2}, \sigma_{3}, \sigma_{4}, \sigma_{5}$ были равны зафиксированным ранее значениям.

Вероятность $R_{1}^{\sigma_{1}, \ldots, \sigma_{5}}\left(\sigma_{0}\right)$ можно вычислить по формуле

$$
R_{1}^{\sigma_{1}, \ldots, \sigma_{5}}\left(\sigma_{0}\right)=\sum_{\gamma_{0}=0_{2}}^{10_{2}} P_{\left(\sigma_{0}, 0,0,0,0,0\right)}^{\left(\gamma_{0}, \sigma_{1}, \ldots, \sigma_{5}\right)}(f, T-s, A)
$$

Таким образом, вычисление искомой вероятности для первого уравнения системы (6) сводится к построению матрицы вероятностей $M(f, A, T-s)$, определенной для уравнения из Теоремы 1. Нас будет интересовать не вся матрица, а лишь ее часть, в которой номера строк будут иметь вид $(*, 0,0,0,0,0)$, где под символом «*» понимается произвольное число, то есть 3 строки. Далее из полученной матрицы надо исключить использование переноса $\gamma_{0}$ : нужно покомпонентно сложить те столбцы матрицы, которые отличаются только переносом $\gamma_{0}$. Таким образом, преобразование матрицы $M(f, A, T-s)$ будет иметь следующий вид:

(1) Исключить все строки, номер которых имеет вид, отличный от $\left(\alpha_{0}, 0,0,0,0,0\right)$.

(2) Сложить все столбцы, номера которых отличаются только в младшем троичном разряде.

В результате будет получена матрица $Q(M(f, A, T-s))=M^{\prime}(f, A, T-s) \in \mathbb{R}^{3 \times 3^{5}}$, элементы которой будут использоваться для анализа первого уравнения системы.

Похожим образом поступим с матрицей для второго уравнения системы (6). $\mathrm{B}$ данной ситуации нас интересуют переносы $\beta_{0}, \beta_{5}$ и все возможные векторы $\alpha$, в которых $\alpha_{0}=\alpha_{5}$. Кроме того, согласно лемме 8 , к вектору параметров данного уравнения будет почленно прибавлен вектор $\left(\beta_{5}, \beta_{1}, \ldots, \beta_{4}\right)$. Таким образом, вторая задача сводится к построению матрицы $N(f, A, s)$ размера $3^{5} \times 3$ : $N(f, A, s)=R\left(M\left(f, A^{[\geqslant T-s]}, s\right)\right)$. В данном случае преобразование матрицы $R$ означает:

(1) Записать в промежуточную матрицу покомпонентные суммы столбцов, совпадающих в младшем и старшем троичных разрядах номера столбца.

(2) Записать в матрицу $N(f, A, s)$ суммы столбцов промежуточной матрицы с совпадающими разностями $\beta_{0}-\beta_{5}$ (когда $\left.\beta_{0}-\beta_{5} \geqslant 0\right)$.

(3) Удалить из матрицы $N(f, A, s)$ строки, для которых $\alpha_{0} \neq \alpha_{5}$.

(4) Переставить строки таким образом, чтобы строки с номером вида $\left(\beta_{0}, \ldots, \beta_{4}\right)$ заняли места строк с номерами вида $\left(\beta_{4}, \beta_{0}, \ldots, \beta_{3}\right)$.

Итак, верна следующая лемма. 
Лемма 9. Вероятность $P(f, T, A)$ можно вычислить по формуле:

$$
P(f, T, A)=\sum_{\left(\sigma_{0}, \ldots, \sigma_{5}\right) \in\left(\left\{0_{2}, 1_{2}, 10_{2}\right\}\right)^{6}} Q\left(M\left(f, A^{[<T-s]}, T-s\right)\right) \cdot R\left(M\left(f, A^{[\geqslant T-s]}, s\right)\right) .
$$

Доказательство. Доказательство леммы приведено выше.

Вернемся к соотношению (14). Верно следующее его представление:

$$
\begin{gathered}
P(f, T, A)=\sum_{\sigma_{0}} \sum_{\left(\sigma_{1}, \ldots, \sigma_{5}\right)}\left(Q\left(M\left(f, A^{[<T-s]}, T-s\right)\right)\right)_{\sigma_{0},\left(\sigma_{1}, \ldots, \sigma_{5}\right)} \times \\
\times\left(R\left(M\left(f, A^{[\geqslant T-s]}, s\right)\right)\right)_{\left(\sigma_{1}, \ldots, \sigma_{5}\right), \sigma_{0}}= \\
=\sum_{\sigma_{0}}\left(Q\left(M\left(f, A^{[<T-s]}, T-s\right)\right) \cdot R\left(M\left(f, A^{[\geqslant T-s]}, s\right)\right)\right)_{\sigma_{0}, \sigma_{0}} .
\end{gathered}
$$

Через $\operatorname{Tr}(M)$, где $M$ - квадратная вещественная матрица, обозначается след матрицы, то есть преобразование, возвращающее сумму диагональных элементов матрицы, переданной на вход. Тогда соотношение (15) может быть записано в виде:

$$
P(f, T, A)=\operatorname{Tr}\left(Q\left(M\left(f, A^{[<T-s]}, T-s\right)\right) \cdot R\left(M\left(f, A^{[\geqslant T-s]}, s\right)\right)\right) .
$$

Воспользуемся формулами для матриц $M(f, A, T)$, построенными в разделе 5.1. Формулу для $P(f, A, T)$ при таких четных значениях величины $T$ и циклического сдвига $s \geqslant 2$, что $T-s \geqslant 2$, можно переписать следующим образом:

$P(f, T, A)=\operatorname{Tr}\left(Q\left(\prod_{i=\frac{T-s}{2}-1}^{0} M\left(f, A^{[i \cdot 2, i \cdot 2+1]}, 2\right)\right) \cdot R\left(\prod_{i=\frac{T}{2}-1}^{\frac{T-s}{2}} M\left(f, A^{[i \cdot 2, i \cdot 2+1]}, 2\right)\right)\right)$.

Формулу для $P(f, A, T)$ при четном значении величины $T$ и таком нечетном значении циклического сдвига $s \geqslant 3$, что $T-s \geqslant 3$, можно переписать следующим образом:

$$
\begin{aligned}
P(f, T, A)= & \operatorname{Tr}\left(Q\left(M\left(f, A^{[T-s-3, T-s-2, T-s-1]}, 3\right) \cdot \prod_{i=\frac{T-s-3}{2}-1}^{0} M\left(f, A^{[i \cdot 2, i \cdot 2+1]}, 2\right)\right) .\right. \\
& \left.\cdot R\left(M\left(f, A^{[T-3, T-2, T-1]}, 3\right) \cdot \prod_{i=\frac{T-3}{2}-1}^{\frac{T-s}{2}} M\left(f, A^{[i \cdot 2, i \cdot 2+1]}, 2\right)\right)\right) \cdot \quad(18)
\end{aligned}
$$

Формулу для $P(f, A, T)$ при нечетном значении величины $T$ и таком четном значении циклического сдвига $s \geqslant 2$, что $T-s \geqslant 3$, можно переписать следующим образом:

$$
\begin{aligned}
P(f, T, A)= & \operatorname{Tr}\left(Q\left(M\left(f, A^{[T-s-3, T-s-2, T-s-1]}, 3\right) \cdot \prod_{i=\frac{T-s-3}{2}-1}^{0} M\left(f, A^{[i \cdot 2, i \cdot 2+1]}, 2\right)\right) .\right. \\
\cdot & \left.R\left(M\left(f, A^{[T-3, T-2, T-1]}, 3\right) \cdot \prod_{i=\frac{T-3}{2}-1}^{\frac{T-s}{2}} M\left(f, A^{[i \cdot 2, i \cdot 2+1]}, 2\right)\right)\right) \cdot \quad(19)
\end{aligned}
$$


Формулу для $P(f, A, T)$ при таких нечетных значениях величины $T$ и циклического сдвига $s \geqslant 3$, что $T-s \geqslant 2$, можно переписать следующим образом:

$$
\begin{aligned}
P(f, T, A)= & \operatorname{Tr}\left(Q\left(\prod_{i=\frac{T-s}{2}-1}^{0} M\left(f, A^{[i \cdot 2, i \cdot 2+1]}, 2\right)\right) .\right. \\
& \left.\cdot R\left(M\left(f, A^{[T-3, T-2, T-1]}, 3\right) \cdot \prod_{i=\frac{T-3}{2}-1}^{\frac{T-s}{2}} M\left(f, A^{[i \cdot 2, i \cdot 2+1]}, 2\right)\right)\right) .
\end{aligned}
$$

В указанных формулах в случае, когда в групповом произведении число множителей равно 0, будем считать произведение равным единичной матрице.

\section{2. Оценки сложности вычислений}

6.2.1. Полный перебор При полном переборе при зафиксированных значениях $A_{j}$ требуется перебрать все варианты значений $X_{i}$, то есть $2^{128}$ вариантов. Общая сложность метода составляет порядка $2^{128}$ подстановок значений $X_{j}$ в уравнение с последующей проверкой равенства правой и левой частей. Сложность одной подстановки -12 арифметических операций. Таким образом, общая сложность $-3 \cdot 2^{129}$.

6.2.2. Предлагаемый метод Общая сложность вычисления по предложенным формулам составляется из сложности вычисления для фиксированной функции $f$ всех матриц $M(f, A, 2)$ и $M(f, A, 3)$, сложности операций перемножения матриц и сложности операций $R, Q$ и $T r$.

Следует отметить, что среди переносов в старшие биты только один может принимать значение, большее 1. Вероятности, соответствующие случаям, когда другие переносы больше 1, всегда равны нулю, поэтому можно исключить из матриц столбцы и строки, соответствующие невозможным значениям. В результате матрицы $M(f, A, 2)$ и $M(f, A, 3)$ можно считать имеющими размер $96 \times 96$ вместо $729 \times 729$.

Сложность вычисления одной матрицы $M(f, A, 2)$ равна

$$
14 \cdot 96 \cdot 96 \cdot 2^{4 \cdot 2}<2^{25}
$$

где 14 - количество операций при проверке одного решения, 96 - число строк и столбцов в матрице, $2^{4 \cdot 2}$ - число вариантов для $X_{i}$.

Поскольку в уравнении участвует 5 параметров $A_{i}$, всего требуется построить $2^{5 \cdot 2}=2^{10}$ матриц. Таким образом, сложность вычисления всех матриц $M(f, A, 2)$ не превосходит $2^{35}$.

Сложность вычисления одной матрицы $M(f, A, 3)$ равна

$$
14 \cdot 96 \cdot 96 \cdot 2^{4 \cdot 3}<2^{29}
$$

где 14 - количество операций при проверке одного решения, 96 - число элементов в матрице, $2^{4 \cdot 3}$ - число вариантов для $X_{i}$.

Поскольку в уравнении участвует 5 параметров $A_{i}$, всего требуется построить $2^{5 \cdot 3}=2^{15}$ матриц. Таким образом, сложность вычисления всех матриц $M(f, A, 3)$ не превосходит $2^{44}$.

Отметим, что вычисленные матрицы $M(f, A, 2)$ и $M(f, A, 3)$ подходят для вычисления вероятности угадывания решения уравнения (2) при любых зафиксированных значениях параметров уравнения $A_{i}$, в то время, как при полном переборе потребуется повторять перебор при каждом новом значении параметров $A_{i}$. 
Сложность перемножения матриц размера $96 \times 96$ не превосходит $96^{3}<2^{20}$. Сложность алгоритмов $R, Q$ и $\operatorname{Tr}$ равна $3^{6}, 3^{6} \cdot 2$ и 3 соответственно.

Общая сложность вычисления вероятности при фиксированных значениях $A_{i}$ и четных $s$ и $T$ составляет

$$
2^{30}+2^{20} \approx 2^{30} \text {. }
$$

Общая сложность вычисления вероятности при фиксированных значениях $A_{i}$ в случае наличия хотя бы одного нечетного числа среди $s$ и $T$

$$
2^{44}+2^{20} \approx 2^{44}
$$

Отметим, что вычисление двух значений вероятности угадывания решения уравнения при разных значениях $A_{i}$ в случае наличия хотя бы одного нечетного числа среди $s$ и $T$ составляет

$$
2^{44}+2 \cdot 2^{20} \approx 2^{44}
$$

а в случае четных $s$ и $T$

$$
2^{30}+2 \cdot 2^{20} \approx 2^{30}
$$

При вычислении матриц $M(f, A, 3)$ в ряде случаев можно по виду параметров $A$ и $\alpha$ установить невозможность получения требуемого значения вектора переносов $\beta$ (например, $A_{1}=0, \alpha_{1}=0, \beta_{1}=1$ ). В этом случае сразу осуществлялся переход к следующим значениям параметров цикла. Данная оптимизация алгоритма позволила добиться уменьшения сложности алгоритма до величины, позволяющей вычислить требуемые вероятности для одной булевой функции и всех значений циклического сдвига за 30 минут на персональной ЭВМ.

Вычисления были проведены для каждой из булевых функций, использованных в конструкции MD5. В результате было установлено, что отношение разностной устойчивости на множестве булевых функций, возникающее при дифференциальном криптоанализе хэш-функции MD5, полностью совпадает с аналогичным отношением, полученным в работе [2]. В частности, булева функция xor является оптимальной с точки зрения сопротивляемости хэш-функции MD5 дифференциальному криптоанализу. При этом значение циклического сдвига не влияет на устойчивость хэш-функции к дифференциальному криптоанализу в рассматриваемой модели.

\section{Список литературы}

1. Biham E., Shamir A., "Differential cryptanalysis of DES-like cryptosystems", J. Cryptology, 4:1 (1991), 3-72.

2. Нгуен Т. Х., Карпунин Г. А., “Оптимальность выбора функции хог в одной модели дифференциального криптоанализа хэш-функций семейства $M D x^{\prime}$, Материалы IV международной научной конференции по проблемам безопасности и противодействия терроризму и VII общероссийской научной конференции «Математика и безопасность информационных технологий» (МАБиТ-2008) (Москва, МГУ, 30 октября -1 ноября 2008г.), 2009, 65-70.

3. Rivest R., "The MD5 message digest algorithm", RFC 1321, MIT Laboratory for Computer Science and RSA Data Security, Inc., April 1992. 